\title{
Changes and Outcome of Cardiac Function in Children with SARS-CoV-2 Multisystem Inflammatory Syndrome
}

\author{
Chloé Huet, MD', Thibault Blache, MD', Thomas Perouse de Montclos, MD ${ }^{1}$, Claire Bertail Galoin, \\ $M D^{1}$, Cécile Leconte, $M D^{1}$, Mohamed Bakloul, MD ${ }^{1}$, Etienne Javouhey, MD, PhD ${ }^{2,3}$, Elodie Perdreau, \\ $M D^{1}$ and Sylvie Di Filippo, $M D, P h D^{1,2^{*}}$
}

${ }^{1}$ Department of Pediatric Cardiology and Congenital Heart Disease, Cardiovascular Hospital Louis Pradel, Hospices Civils de Lyon, Lyon, France

${ }^{2}$ University of Lyon Medical Center, Claude Bernard University Lyon 1, Lyon, France

${ }^{3}$ Department of Pediatric Intensive Care, Femme-Mère-Enfant Hospital, Hospices Civils de Lyon, France

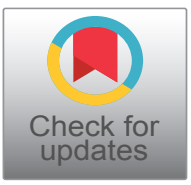

*Corresponding author: Sylvie Di Filippo, MD, PhD, Department of Pediatric Cardiology and Congenital Heart Disease, Cardiovascular Hospital Louis Pradel, Hospices Civils de Lyon, Lyon; University of Lyon Medical Center, Claude Bernard University Lyon 1, Lyon, France, Tel: +33-610-321-512, Fax: +33-472-357-049

\section{Abstract}

Background: Cardiac involvement has been reported in children with SARS-CoV-2 inflammatory syndrome. The objectives herein were to assess changes in cardiac parameters and compare cardiac pathologies observed in SARS-CoV-2 to Kawasaki-(KW) and myocarditis-(MY).

Methods: Patients $<18$ years of age, with symptoms of Kawasaki disease (KW group) and/or of acute myocarditis (MY group) from March 1 to May 312020 were included. Clinical, laboratory data, and cardiac parameters were recorded upon diagnosis and at several points over two weeks.

Results: Sixteen patients ( 8 males) diagnosed in the context of SARS-CoV-2 pandemic, were included ( $8 \mathrm{KW}, 8$ $\mathrm{MY}$ ); the mean age was 5.9 years (KW 2.7 years, MY 10.2 years; $p=0.0002)$. Fever was present in all. Conjunctivitis, buccal inflammation, lymphadenopathies, dermatological lesions were more frequent in $\mathrm{KW}$, chest pain, and gastrointestinal symptoms more frequent in MY. C-reactive protein, procalcitonin, and fibrinogen levels were higher in MY. Platelets were higher, and hemoglobin and hematocrit lower in $\mathrm{KW}$. Troponin and NT pro-BNP were higher in MY. Coronary anomalies were observed in $\mathrm{KW}$, and mitral regurgitation and pericarditis in MY. LV systolic function impairment in $M Y$ was concomitant to the highest values of troponin, NT-pro-BNP, CRP, ferritin, D-dimers, and lowest value of lymphocytes and platelets. Mean length of intensive care stay was 7.8 days and that of hospital stay was 9.7 days (MY 5.1 days, KW 14.4 days, $p=0.0008$ ). Systolic function
\end{abstract}

was normalized within 15 days. Diastolic dysfunction appeared after normalization of systolic function.

Conclusion: Cardiac injury is parallel to biological changes, as evidenced by serial changes of systolic and diastolic myocardial ultrasound parameters.

\section{Keywords}

SARS-CoV-2, Myocarditis, Kawasaki disease, Echocardiography, Inflammatory syndrome, Cardiac function

\section{Introduction}

SARS-CoV-2 infection (COVID-19) has spread rapidly worldwide. While during the first wave of the pandemic children were not severely affected, a late wave unexpectedly occurred and pediatric patients presented with a new multisystem inflammatory disease mimicking Kawasaki disease (KW) and/or acute myocarditis (MY). Previous publications have reported the clinical presentation, laboratory data, and short-term outcomes of such cases to describe this novel pediatric syndrome triggered by SARS-CoV-2 [1-6]. To our knowledge, repeated measures of laboratory and of concomitant cardiac parameters have not been previously assessed in these pediatric cases, whereas cardiac data have been reported in adults [7-12].

Citation: Huet C, Blache T, Montclos TP, Galoin CB, Leconte C, et al. (2020) Changes and Outcome of Cardiac Function in Children with SARS-CoV-2 Multisystem Inflammatory Syndrome. Int J Clin Cardiol 7:210. doi.org/10.23937/2378-2951/1410210

Accepted: December 24, 2020: Published: December 26, 2020

Copyright: (C) 2020 Huet C, et al. This is an open-access article distributed under the terms of the Creative Commons Attribution License, which permits unrestricted use, distribution, and reproduction in any medium, provided the original author and source are credited. 
We report herein the experience of the Lyon (France) medical center for children diagnosed from March to the end of May 2020, and compare data to that reported in the literature. The objectives of the study were to describe the clinical, laboratory, and cardiac data of pediatric patients suffering from SARS-CoV-2 multisystem inflammatory disease, to compare the myocarditis-like to Kawasaki-like cases, to evaluate the changes over time in the early phase the disease with repeated measurements of laboratory and cardiac parameters, and to evaluate short-term outcomes.

\section{Methods}

All patients under the age of 18 , who were admitted during the SARS-CoV- 2 pandemic at the Lyon medical center from March 1 to May 31, 2020, with fever and symptoms of KW and/or hemodynamic failure (MY), were included in the cohort. The SARS-CoV-2 was proven by PCR result or familial at-risk contact, or was suspected in the context of the epidemic period.

Hemodynamic failure was defined as compromised organ tissue perfusion, hypotension, tachycardia, signs of heart failure, low cardiac output, oliguria and inotrope support requirement, due to impaired myocardial function.

The following laboratory data were recorded at hospitalization and at different time points during hospital stay: C-reactive protein, procalcitonin, hemoglobin, hematocrit, leukocyte count, neutrophil count, lymphocyte count, platelet count, albumin, ferritin, D-dimers, fibrinogen, NT pro-BNP, and troponin; the time points were: at diagnosis: Day-0, day-1-2, day-3,day-4, day-5, day-6, day-8, day-10, day-12, and day-14.

The patients were divided into MY and KW groups and compared with regard to laboratory data and echocardiographic parameters. The MY group included cases with symptoms of heart failure and/or compromised hemodynamics and impaired myocardial function by ultrasound examination; the KW group included patients with clinical symptoms of KW and preserved myocardial function.

Cardiac function was evaluated by echocardiography and Doppler. Ultrasound assessment of left ventricle (LV) systolic function included measurements of ventricular end-diastolic ventricular diameter (LVEDD), ventricular end-systolic ventricular diameter (LVESD), left ventricular ejection fraction (LVEF) using the Simpson method, left ventricular shortening fraction calculated as (LVEDD-LVESD)/LVEDD and sub-aortic velocity integral (VTI), of right ventricular function by tricuspid annular plane systolic excursion (TAPSE), of LV diastolic function by $E$ and $A$ mitral waves, E/A ratio, and Doppler tissue imaging indices including Ea wave at mitral annulus and E/Ea ratio. Mitral regurgitation, pericardial effusion and coronary arteries anomalies were also assessed.
Cardiac ultrasound data were recorded at admission and at each time-point defined above and compared to simultaneous laboratory data.

LVEF was considered as impaired if $<60 \%$ and LVSF if $<35 \%$.

\section{Statistical Analysis}

All data were entered into an Excel spreadsheet (Microsoft Excel 97-2003, Redmond, WA, USA), and Statview software version 5.0 (SAS Institute Inc., Cary, NC, USA) was used for statistical analysis. Continuous variables were expressed as mean \pm standard deviation (SD), and categorical variables as counts and percentage. Comparisons were made using the two-tailed paired Student's t test or the Mann-Whitney

$\mathrm{U}$ test for the continuous variables, and the $\mathrm{Chi}^{2}$ test for nominal variables. The level of statistical significance was set at a $p$ value of less than 0.05 .

\section{Results}

Sixteen patients were included, 8 males and 8 females, and the mean \pm SD age was $5.9 \pm 4.6$ years (range: 2.5 months -3.2 years) at diagnosis. Among these, 8 were included in the MY group, and 8 in the KW group. The mean age at diagnosis was significantly lower in $\mathrm{KW}$ group (mean \pm standard deviation $=2.7 \pm 2.0$ years) than in the MY group $(10.3 \pm 3.3$ years; $p=0.0002)$, as were weight and body mass index at diagnosis (Table 1 ).

An at-risk contact with a family relative infected with COVID-19 was proved in 6 cases (37.5\%): 2 in KW and 4 in MY group, and PCR swab was COVID-19 positive in 4 cases (25.0\%): 0 in KW and 4 in MY group (Table 1). Onset of symptoms occurred at mean \pm standard deviation $=4.7 \pm 2.4$ days prior to diagnosis. Fever was present in all cases. Dermatological lesions were significantly more frequent in the KW group and there was a trend towards more frequent conjunctivitis, buccal mucosal inflammation (cheilitis), and lymphadenopathies in this group, while there was a trend towards more frequent chest pain, gastrointestinal, and respiratory symptoms in the MY group; the frequency of neurological and ENT symptoms at onset did not differ significantly between both groups (Table 1 ). A total of $6 / 8$ patients in the MY group presented with clinical symptoms of severe heart failure and were admitted to the intensive care unit at the time of diagnosis, whereas cardiac function deteriorated within 2 days after admission in the other 2 cases of MY group.

Lymphocyte counts were significantly lower in the $\mathrm{MY}$ group, while platelet counts were significantly higher and hemoglobin and hematocrit were significantly lower in the KW group. There was a trend towards higher C-reactive protein, procalcitonin, and fibrinogen levels in the MY group. D-dimers, ferritin, troponin, and NT-proBNP levels were elevated in MY patients (Table 2). 
Table 1: Patient characteristics.

\begin{tabular}{|c|c|c|c|c|}
\hline & $\begin{array}{l}\text { Total } \\
N=16\end{array}$ & $\begin{array}{l}\mathrm{KW} \text { group } \\
\mathbf{N}=\mathbf{8}\end{array}$ & $\begin{array}{l}\text { MY group } \\
\mathbf{N}=\mathbf{8}\end{array}$ & $\mathbf{p}$ \\
\hline $\begin{array}{l}\text { Sex, } \mathrm{n}(\%) \\
\text { Male } \\
\text { Female }\end{array}$ & $\begin{array}{l}8(50.0) \\
8(50.0)\end{array}$ & $\begin{array}{l}4(50.0) \\
4(50.0)\end{array}$ & $\begin{array}{l}4(50.0) \\
4(50.0)\end{array}$ & - \\
\hline $\begin{array}{l}\text { Age at diagnosis (years) } \\
\text { mean } \pm \text { standard deviation } \\
\text { range }\end{array}$ & $\begin{array}{l}5.9 \pm 4.6 \\
0.15-13.2\end{array}$ & $\begin{array}{l}2.7 \pm 2.0 \\
0.15-5.8\end{array}$ & $\begin{array}{l}10.3 \pm 3.3 \\
4.0-13.2\end{array}$ & 0.0002 \\
\hline $\begin{array}{l}\text { Weight at diagnosis }(\mathrm{kg}) \\
\text { mean } \pm \text { standard deviation } \\
\text { range }\end{array}$ & $\begin{array}{l}26.1 \pm 20.2 \\
4.5-60.0\end{array}$ & $\begin{array}{l}12.6 \pm 5.6 \\
4.5-23.4\end{array}$ & $\begin{array}{l}44.1 \pm 18.6 \\
14.9-60.0\end{array}$ & 0.006 \\
\hline $\begin{array}{l}\text { Body mass index }\left(\mathrm{kg} / \mathrm{m}^{2}\right) \\
\text { mean } \pm \text { standard deviation } \\
\text { range }\end{array}$ & $\begin{array}{l}17.1 \pm 3.5 \\
13.3-24.0\end{array}$ & $\begin{array}{l}15.6 \pm 1.8 \\
13.3-19.2\end{array}$ & $\begin{array}{l}19.0 \pm 4.5 \\
13.3-24.0\end{array}$ & 0.09 \\
\hline COVID at-risk contact, $\mathrm{n}(\%)$ & $6(37.5)$ & $2(25.0)$ & $4(50.0)$ & 0.33 \\
\hline PCR-positive COVID, n (\%) & $4(25.0)$ & $0(0.0)$ & $4(50.0)$ & 0.024 \\
\hline $\begin{array}{l}\text { Onset of symptoms to diagnosis (days) } \\
\text { mean } \pm \text { standard deviation } \\
\text { range }\end{array}$ & $\begin{array}{l}4.7 \pm 2.4 \\
1.0-9.0\end{array}$ & $\begin{array}{l}5.5 \pm 2.5 \\
2.0-9.0\end{array}$ & $\begin{array}{l}3.8 \pm 2.0 \\
1.0-7.0\end{array}$ & 0.21 \\
\hline Fever, n (\%) & $14(100.0)$ & $8(100.0)$ & $8(100.0)$ & - \\
\hline Dermatological signs, n (\%) & $9(64.3)$ & $7(87.5)$ & $2(25.0)$ & 0.036 \\
\hline Conjunctivitis, n (\%) & $8(50.0)$ & $5(62.5)$ & $3(37.5)$ & 0.28 \\
\hline Cheilitis, n (\%) & $8(50.0)$ & $5(62.5)$ & $3(37.5)$ & 0.28 \\
\hline Lymphadenopathies, n (\%) & $11(68.7)$ & $7(87.5)$ & $4(50.0)$ & 0.12 \\
\hline Chest pain, n (\%) & $3(18.7)$ & $0(0.0)$ & $3(37.5)$ & 0.07 \\
\hline Gastrointestinal symptoms, n (\%) & $10(64.3)$ & $4(50.0)$ & $6(75.0)$ & 0.19 \\
\hline Respiratory symptoms, n (\%) & $4(25)$ & $1(12.5)$ & $3(37.5)$ & 0.12 \\
\hline Neurological symptoms, n (\%) & $4(28.5)$ & $2(25.0)$ & $2(25.0)$ & 0.73 \\
\hline ENT symptoms, n (\%) & $9(64.3)$ & $5(62.5)$ & $4(50)$ & 0.87 \\
\hline $\begin{array}{l}\text { Intravenous immunoglobulins, } \mathrm{n}(\%) \\
1 \text { dose } 2 \mathrm{~g} / \mathrm{kg} \\
2 \text { doses } 1 \mathrm{~g} / \mathrm{kg} / \text { dose }\end{array}$ & $\begin{array}{l}9(64.3) \\
7(35.7)\end{array}$ & $\begin{array}{l}8(100.0) \\
0(0.0)\end{array}$ & $\begin{array}{l}1(16.7) \\
6(83.3)\end{array}$ & 0.0013 \\
\hline
\end{tabular}

ENT: Ear Nose Throat

Table 2: Laboratory data at admission.

\begin{tabular}{|c|c|c|c|c|}
\hline & $\begin{array}{l}\text { Total } \\
N=16\end{array}$ & $\begin{array}{l}\mathrm{KW} \text { group } \\
\mathrm{N}=8\end{array}$ & $\begin{array}{l}\text { MY group } \\
N=8\end{array}$ & $\mathbf{p}$ \\
\hline $\begin{array}{l}\text { Lymphocyte count }\left(10^{3} / \mathrm{mL}\right) \\
\text { Mean } \pm \text { standard deviation } \\
\text { range }\end{array}$ & $\begin{array}{l}2.3 \pm 2.1 \\
0.35-6.8\end{array}$ & $\begin{array}{l}3.3 \pm 2.8 \\
0.71-6.8\end{array}$ & $\begin{array}{l}0.8 \pm 0.3 \\
0.35-1.3\end{array}$ & 0.019 \\
\hline $\begin{array}{l}\text { Platelet count }\left(10^{3} / \mathrm{mL}\right) \\
\text { mean } \pm \text { standard deviation } \\
\text { range }\end{array}$ & $\begin{array}{l}268.0 \pm 178.0 \\
115.0-753.0\end{array}$ & $\begin{array}{l}349.0 \pm 202.0 \\
115.0-753.0\end{array}$ & $\begin{array}{l}159.0 \pm 22.0 \\
128.0-192.0\end{array}$ & 0.042 \\
\hline $\begin{array}{l}\text { Hemoglobin }(\mathrm{g} / \mathrm{L}) \\
\text { mean } \pm \text { standard deviation } \\
\text { range }\end{array}$ & $\begin{array}{l}115.5 \pm 14.0 \\
95.0-145.0\end{array}$ & $\begin{array}{l}109.2 \pm 10.3 \\
95.0-128.0\end{array}$ & $\begin{array}{l}123.8 \pm 14.6 \\
100.0-145.0\end{array}$ & 0.049 \\
\hline $\begin{array}{l}\text { Hematocrit }(\%) \\
\text { mean } \pm \text { standard deviation } \\
\text { range }\end{array}$ & $\begin{array}{l}34.1 \pm 3.4 \\
28.3-41.0\end{array}$ & $\begin{array}{l}32.3 \pm 2.4 \\
28.3-36.1\end{array}$ & $\begin{array}{l}36.2 \pm 3.4 \\
31.0-41.0\end{array}$ & 0.033 \\
\hline $\begin{array}{l}\text { Leucocyte count }\left(10^{3} / \mathrm{mL}\right) \\
\text { mean } \pm \text { standard deviation } \\
\text { range }\end{array}$ & $\begin{array}{l}13.2 \pm 6.5 \\
5.4-25.8\end{array}$ & $\begin{array}{l}13.9 \pm 7.6 \\
6.6-25.8\end{array}$ & $\begin{array}{l}12.1 \pm 5.3 \\
5.4-20.2\end{array}$ & 0.634 \\
\hline
\end{tabular}




\begin{tabular}{|c|c|c|c|c|}
\hline $\begin{array}{l}\text { Neutrophil count }\left(10^{3} / \mathrm{mL}\right) \\
\text { mean } \pm \text { standard deviation } \\
\text { range }\end{array}$ & $\begin{array}{l}9.3 \pm 4.6 \\
2.4-17.2\end{array}$ & $\begin{array}{l}8.5 \pm 4.4 \\
2.4-16.5\end{array}$ & $\begin{array}{l}10.2 \pm 4.9 \\
4.5-17.2\end{array}$ & 0.518 \\
\hline $\begin{array}{l}\text { C-reactive protein }(\mathrm{mg} / \mathrm{L}) \\
\text { mean } \pm \text { standard deviation } \\
\text { range }\end{array}$ & $\begin{array}{l}206.0 \pm 104.2 \\
50.0-448.0\end{array}$ & $\begin{array}{l}160.5 \pm 78.4 \\
50.0-312.6\end{array}$ & $\begin{array}{l}266.7 \pm 109.1 \\
121.0-448.0\end{array}$ & 0.054 \\
\hline $\begin{array}{l}\text { Procalcitonin }(\mathrm{ng} / \mathrm{mL}) \\
\text { mean } \pm \text { standard deviation } \\
\text { range }\end{array}$ & $\begin{array}{l}54.6 \pm 85.1 \\
1.2-218.0\end{array}$ & $\begin{array}{l}14.7 \pm 17.9 \\
2.0-27.4\end{array}$ & $\begin{array}{l}74.5 \pm 101.8 \\
1.2-218.0\end{array}$ & 0.479 \\
\hline $\begin{array}{l}\text { Fibrinogen }(\mathrm{g} / \mathrm{L}) \\
\text { mean } \pm \text { standard deviation } \\
\text { range }\end{array}$ & $\begin{array}{l}7.1 \pm 2.4 \\
3.0-9.6\end{array}$ & $\begin{array}{l}5.9 \pm 2.7 \\
3.0-9.3\end{array}$ & $\begin{array}{l}8.4 \pm 1.1 \\
7.5-9.6\end{array}$ & 0.208 \\
\hline $\begin{array}{l}\text { Albumin }(\mathrm{g} / \mathrm{L}) \\
\text { mean } \pm \text { standard deviation } \\
\text { range }\end{array}$ & $\begin{array}{l}32.5 \pm 8.9 \\
21.0-42.0\end{array}$ & - & $\begin{array}{l}32.5 \pm 8.9 \\
21.0-42.0\end{array}$ & - \\
\hline $\begin{array}{l}\text { D-dimers }(\mathrm{ng} / \mathrm{mL}) \\
\text { mean } \pm \text { standard deviation } \\
\text { range }\end{array}$ & $\begin{array}{l}4096.2 \pm 2754.3 \\
283.0-7223.0\end{array}$ & $283.0^{*}$ & $\begin{array}{l}5049.5 \pm 2014.1 \\
2737.0-7223.0\end{array}$ & - \\
\hline $\begin{array}{l}\text { Ferritin }(\mathrm{ng} / \mathrm{mL}) \\
\begin{array}{l}\text { mean } \pm \text { standard deviation } \\
\text { range }\end{array}\end{array}$ & $\begin{array}{l}693.2 \pm 306.7 \\
300.0-1036.0\end{array}$ & 1036.0* & $\begin{array}{l}579.0 \pm 250.6 \\
300.0-785.0\end{array}$ & - \\
\hline $\begin{array}{l}\text { Troponin }(\mathrm{ng} / \mathrm{L}) \\
\text { mean } \pm \text { standard deviation } \\
\text { range }\end{array}$ & $\begin{array}{l}1440.4 \pm 4011.3 \\
5.0-12123.0\end{array}$ & $\begin{array}{l}24.7 \pm 20.2 \\
13.0-55.0\end{array}$ & $\begin{array}{l}2573.0 \pm 5345.4 \\
5.0-12123.0\end{array}$ & 0.378 \\
\hline $\begin{array}{l}\text { NT pro-BNP }(\mathrm{pg} / \mathrm{mL}) \\
\text { mean } \pm \text { standard deviation } \\
\text { range }\end{array}$ & $\begin{array}{l}5050.5 \pm 10184.2 \\
53.0-25796.0\end{array}$ & $805.0^{*}$ & $\begin{array}{l}5899.6 \pm 11146.3 \\
53.0-25796.0\end{array}$ & - \\
\hline
\end{tabular}

"one patient tested

Table 3: Echocardiographic data at admission.

\begin{tabular}{|c|c|c|c|c|}
\hline & $\begin{array}{l}\text { Total } \\
N=16\end{array}$ & $\begin{array}{l}\text { KW group } \\
N=8\end{array}$ & $\begin{array}{l}\text { MY group } \\
N=8\end{array}$ & $\mathbf{p}$ \\
\hline Coronary artery abnormalities, $\mathrm{n}(\%)$ & $5(31.2)$ & $4(50.0)$ & $1(6.2)$ & 0.040 \\
\hline $\begin{array}{l}\text { LVEF }(\%) \\
\text { mean } \pm \text { standard deviation } \\
\text { range }\end{array}$ & $\begin{array}{l}64.3 \pm 12.4 \\
38.0-83.0\end{array}$ & $\begin{array}{l}71.5 \pm 7.8 \\
66.0-83.0\end{array}$ & $\begin{array}{l}58.5 \pm 11.6 \\
38.0-60.0\end{array}$ & 0.088 \\
\hline LVEF $<60 \%, \mathrm{n}(\%)$ & $6(37.5)$ & $0(0.0)$ & $6(75.0)$ & 0.0019 \\
\hline 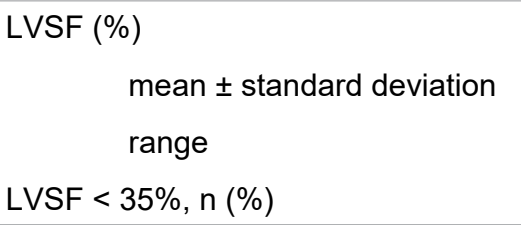 & $\begin{array}{l}35.2 \pm 7.7 \\
18.0-49.7 \\
6(37.5)\end{array}$ & $\begin{array}{l}38.1 \pm 5.8 \\
35.0-49.7 \\
0(0.0)\end{array}$ & $\begin{array}{l}30.8 \pm 8.6 \\
18.0-34.0 \\
6(75.0)\end{array}$ & $\begin{array}{l}0.108 \\
.0019\end{array}$ \\
\hline Mitral regurgitation, $\mathrm{n}(\%)$ & $7(43.7)$ & $1(12.5)$ & $6(75.0)$ & 0.008 \\
\hline Pericarditis, n (\%) & $5(31.2)$ & $1(12.5)$ & $4(50.0)$ & 0.124 \\
\hline $\begin{array}{l}\text { TAPSE }(\mathrm{mm}) \\
\qquad \begin{array}{l}\text { mean } \pm \text { standard deviation } \\
\text { range }\end{array}\end{array}$ & $\begin{array}{l}17.1 \pm 4.0 \\
10.0-21.0\end{array}$ & $\begin{array}{l}17.0 \pm 4.2 \\
10.0-21.0\end{array}$ & $\begin{array}{l}17.5 \pm 4.5 \\
11.0-21.0\end{array}$ & 0.933 \\
\hline $\begin{array}{l}\text { LVEDD } / \mathrm{m}^{2}\left(\mathrm{~mm} / \mathrm{m}^{2}\right) \\
\text { mean } \pm \text { standard deviation } \\
\text { range }\end{array}$ & $\begin{array}{l}43.3 \pm 12.9 \\
24.2-61.6\end{array}$ & $\begin{array}{l}53.3 \pm 5.5 \\
45.9-61.6\end{array}$ & $\begin{array}{l}33.3 \pm 9.7 \\
24.2-51.5\end{array}$ & 0.0014 \\
\hline
\end{tabular}




\begin{tabular}{|c|c|c|c|c|}
\hline $\begin{array}{l}\operatorname{LVESD} / \mathrm{m}^{2}\left(\mathrm{~mm} / \mathrm{m}^{2}\right) \\
\text { mean } \pm \text { standard deviation } \\
\text { range }\end{array}$ & $\begin{array}{l}28.4 \pm 6.2 \\
17.4-35.0\end{array}$ & $\begin{array}{l}32.9 \pm 1.6 \\
31.5-35.0\end{array}$ & $\begin{array}{l}22.9 \pm 4.9 \\
17.4-29.2\end{array}$ & 0.0012 \\
\hline $\begin{array}{l}\text { E/A ratio } \\
\qquad \begin{array}{l}\text { mean } \pm \text { standard deviation } \\
\text { range }\end{array}\end{array}$ & $\begin{array}{l}1.8 \pm 0.9 \\
1.0-3.6\end{array}$ & $\begin{array}{l}1.6 \pm 0.3 \\
1.2-1.8\end{array}$ & $\begin{array}{l}2.1 \pm 1.3 \\
1.0-3.6\end{array}$ & 0.566 \\
\hline $\begin{array}{l}\text { E/Ea ratio } \\
\qquad \begin{array}{l}\text { mean } \pm \text { standard deviation } \\
\text { range }\end{array}\end{array}$ & $\begin{array}{l}9.0 \pm 1.7 \\
7.2-11.1\end{array}$ & - & $\begin{array}{l}9.0 \pm 1.7 \\
7.2-11.1\end{array}$ & \\
\hline
\end{tabular}

LVEDD: Left Ventricular End-Diastolic Diameter; LVEF: Left Ventricle Ejection Fraction; LVESD: Left Ventricular End-Systolic Diameter; LVSF: Left Ventricle Shortening Fraction; TAPSE: Tricuspid Annular Plane Systolic Excursion
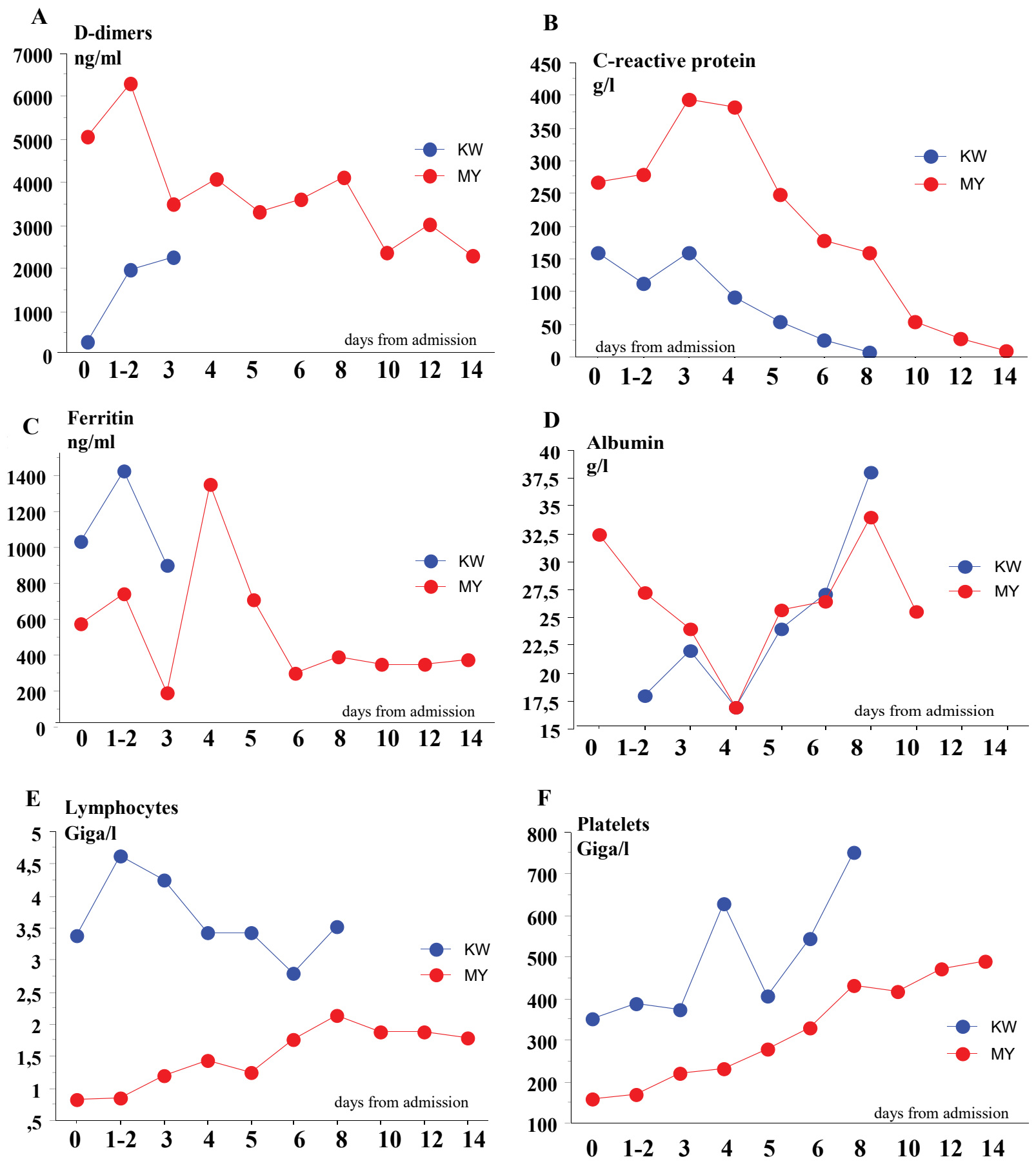

Figure 1: Laboratory parameters blood levels changes over the early phase of COVID 19. (A) D-dimers; (B) C-reactive protein; (C) Ferritin; (D) Albumin; (E) Lymphocytes counts; (F) Platelet counts. 
Coronary artery abnormalities were observed in 5 cases (31.2\%): $4 \mathrm{KW}$ patients (50.0\%), $1 \mathrm{MY}$ patient (6.2\%). No coronary aneurysm was diagnosed, coronary artery dilation was the only observed coronary artery lesion. One patient in MY group exhibited dilatation of right and left coronary arteries. Myocardial systolic function was impaired in the MY group (LVEF < 60\%, LVSF $<35 \%$ ). Mitral regurgitation was significantly more frequent, and there was a trend towards this for pericarditis in the MY group. Right ventricular systolic function was preserved (as assessed by TAPSE index) and did not differ significantly between both groups. Indexed left ventricular end-diastolic diameters were within the normal range in the MY group whereas end-systolic diameters were increased. LV diameters were significantly greater in the KW group than in the MY group. There was no significant difference between groups regarding the indices of diastolic function at diagnosis (E/A and E/ Ea ratios) at presentation (Table 3 ).

\section{Outcomes}

All patients received a $2 \mathrm{~g}$ per $\mathrm{kg}$ dose of intravenous immunoglobulins infusion within 24 hours after admission (at day- 0 or day-1-2): A single dose in the $\mathrm{KW}$ group and two doses of $1 \mathrm{~g} / \mathrm{kg} /$ day each (for 2 days) in the MY group. Inotrope support was needed in $6 / 8$ patients of the MY group (the duration of which ranged from 4 to 7 days, mean 5 days) and none of the KW group. No patient needed extracorporeal membrane oxygenator (ECMO) support. Other therapeutic management included: interleukin 1 antagonist monoclonal antibody in 2 cases ( $\mathrm{KW}$ group), and corticosteroids in 2 cases. The mean \pm standard deviation duration of intensive care stay was $7.8 \pm 5.8$ days in MY patients (no KW patient was admitted to an intensive care unit). The mean \pm standard deviation duration of hospital stay was $9.7 \pm$ 6.1 days; it was longer in the MY group (5.1 \pm 3.5 days) than in the $\mathrm{KW}$ group $(14.4 \pm 4.2$ days; $p=0.0008)$.

Mean \pm standard deviation levels of D-dimers, C-reactive protein and ferritin (Figure $1 \mathrm{~A}$, Figure $1 \mathrm{~B}$ and Figure $1 C$ ) were elevated from admission and peaked respectively at day 1-2, day 3 and day 4; they were higher in MY than KW patients. In both groups, the mean albumin levels were generally below normal values and lowest levels occurred at day 4 (Figure 1D). Lymphopenia and thrombopenia were observed in MY patients at admission (day 0) and normalized progressively during the first five days; lymphocyte count was within the normal range, and platelet count increased beyond fifth day in KW patients (Figure 1E and Figure 1F)).

A qualitative serological test was performed in all patients. Anti-SARS-CoV-2 IgG antibodies were positive in $7 / 8$ patients in the $M Y$ group (87.5\%) and none in $\mathrm{KW}$ group.

\section{Cardiac function in the MY group}

LV systolic function (assessed by LVEF, LVSF) was impaired at onset and decreased within the first 4 days after admission, the lowest value was recorded at day 1-2 and day 3 (Figure $2 \mathrm{~A}$ and Figure $2 \mathrm{~B}$ ), concomitant to the highest levels of troponin and NT-pro-BNP (Figure $2 \mathrm{C}$ and Figure 2D), and to peak levels of D-dimers, C-reactive protein, ferritin, and nadir levels of lymphocyte and platelets counts (Figure $1 \mathrm{~A}$ to Figure $1 \mathrm{C}$, Figure $1 \mathrm{E}$ and Figure $1 \mathrm{~F}$ ). Ultrasound parameters of systolic function returned to normal values within 10 days after admission. Diastolic dysfunction, identified by increased $E / A$ and $E / E$ a ratios, was observed in the immediate course after normalization of systolic function, i.e. from T8 (day 14; Figure 2E and Figure 2F).

All patients in the MY group received angiotensin-convertor enzyme inhibitors and a first dose of beta blockers associated with aspirin antiplatelet therapy prior to discharge home.

\section{Discussion}

The pediatric SARS-CoV-2 cardiovascular disease was characterized by severe inflammation (evidenced by CRP and fibrinogen) with decreased albumin levels, lymphopenia, and elevated D-dimers and ferritin blood levels, as has been reported elsewhere [1-6]. Some investigators have suggested that the coronavirus family might be one of the triggers of $\mathrm{KW}$, SARS-CoV-2 being a particularly virulent strain able to elicit a powerful immune response in the host [13-18]. Acute MY causing severe left ventricle dysfunction has also been reported in the SARS-CoV-2 pandemic and might result from the same mechanisms [19-21]. The two clinical and laboratory test profiles, namely $\mathrm{KW}$ and $\mathrm{MY}$, might be various expressions of the same disease. In the present study, all patients suffered from multisystem inflammatory syndrome which was the most severe in the MY group; the MY-type cases are characterized by a more severe laboratory test profile than the KW-type cases. One notable clinical feature is the frequency of gastrointestinal symptoms which can imitate severe acute peritonitis, so that some patients undergo laparoscopy for misdiagnosed peritonitis due to appendicitis; this was the case for one patient herein, and has been reported elsewhere [19].

The present study aimed to analyze the changes over time in myocardial function during the early phase of the disease and to assess the concomitant variations of laboratory test parameters and cardiac biomarkers. We found that myocardial systolic function is altered early during the inflammatory reaction; decreased ejection fraction was parallel to the peak of NTpro-BNP and occurred shortly after the troponin peak blood level. Thus, troponin, as a biomarker of myocardial injury, might help to predict cardiac dysfunction in such patients.

The prompt improvement of systolic function after intravenous immunoglobulin infusion, is in favor of reversible myocardial lesions. The mechanism of $\mathrm{CO}$ - 
A
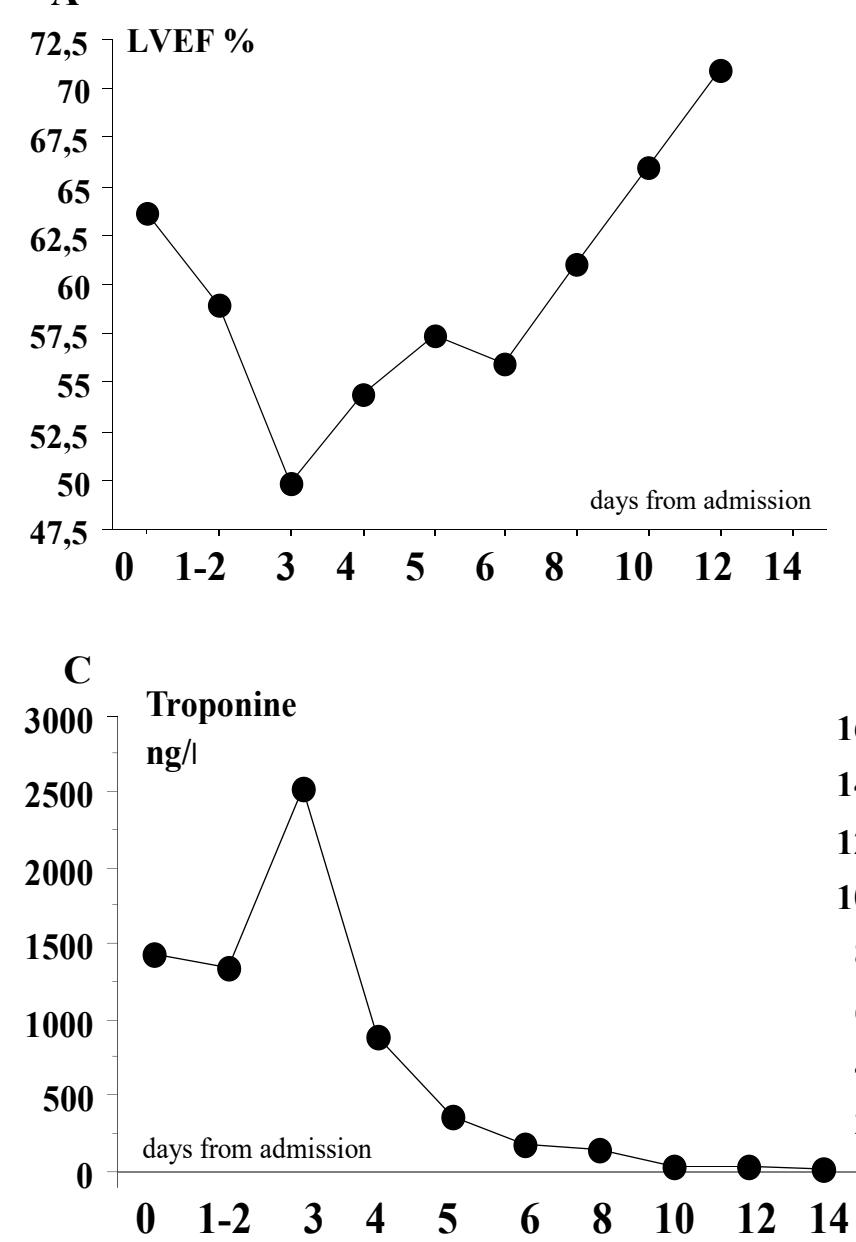
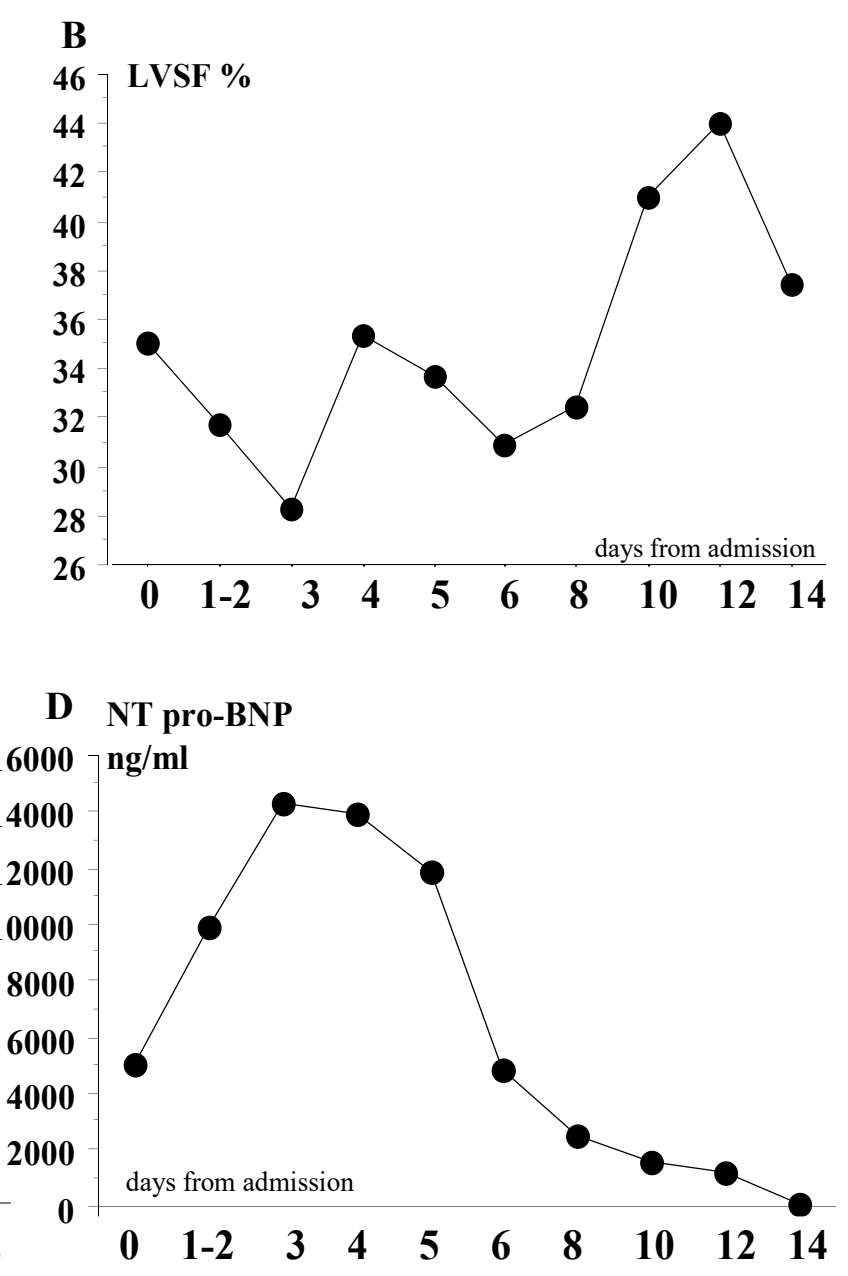
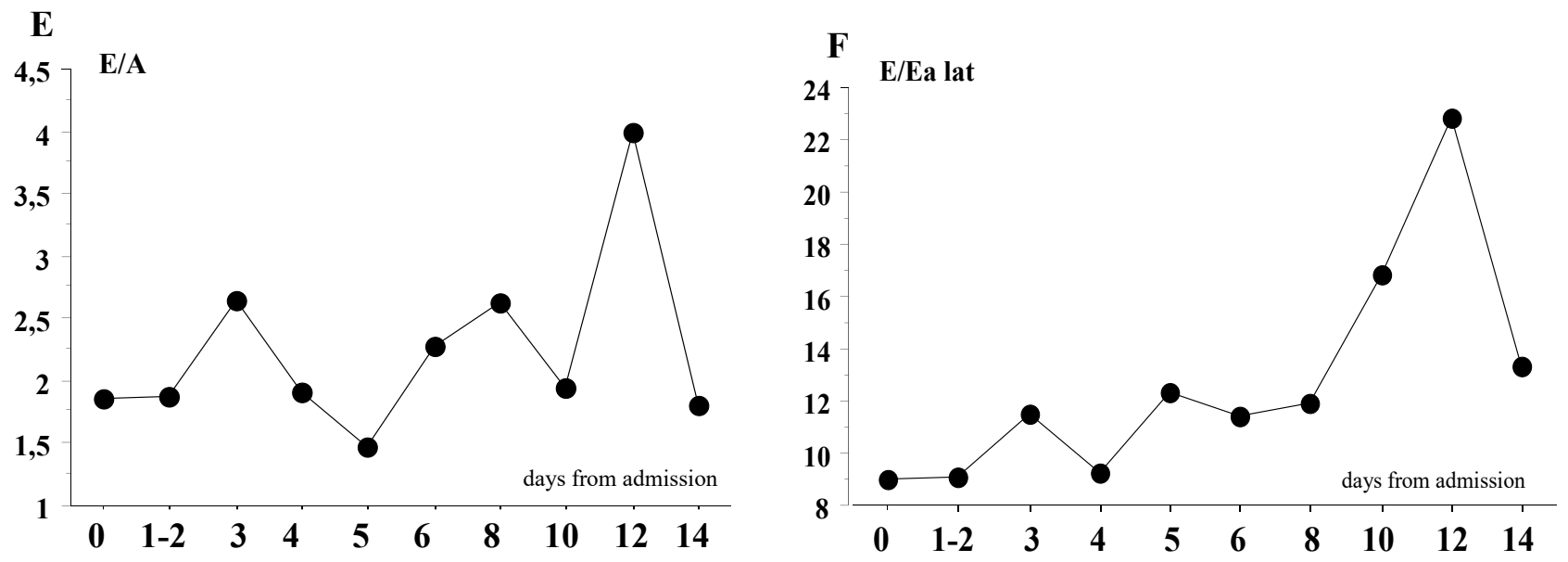

Figure 2: Myocardial ultrasound parameters of systolic function (LVSF, LVEF), diastolic function (E/A ratio, E/Ealat and cardiac biomarkers blood levels (troponin and NT pro-BNP) changes over the early phase of COVID 19. (A) LVSF (Left Ventricle Shortening Fraction); (B) LVEF (Left Ventricle Ejection Fraction); (C) Trponine; (D) NT pro-BNP; (E) Ratio E/A (mitral pulsed Doppler E wave and A wave); (F) Ratio E/Ea (mitral pulsed Doppler E wave and tissue Doppler imaging at mitral annulus Ea wave).

VID-19 -induced MY is still a matter of debate and is supposed to be of multifactorial origin, i.e. direct viral injury, cytokine storm and myocardial hypoxemia [2022]. In this model, SARS-CoV-2 leads to an inflammatory state, which results in a cytokine storm triggered by an uncontrolled and dysfunctional immune response [22-24]; cytokines then cause apoptosis and necrosis of myocardial cells, and viral infection may induce incre- ased myocardial oxygen demand and create a state of oxygen supply-demand mismatch [22-25]. Aggression of the myocardium alters systolic function parallel to the biological inflammatory state. Direct viral attack on the myocardium is unlikely since the symptomatology is delayed by almost a month compared to the infectious contact and the positivity of nasal PCR is rare, which indicates low viral load at this stage. It is most likely that 
the inflammatory aggression of the myocardium is due to activation of the post-viral cytokine inflammatory cascade; this mechanism is comparable to $\mathrm{KW}$. The major cytokine inflammation responsible for dramatic fall in systemic vascular resistance, might also lead to additional myocardial ischemia aggravating the process of acute myocardial aggression. This is supported by the requirement of norepinephrine to stabilize compromised hemodynamics in these patients, both in our experience and others' $[26,27]$. The rise in troponin occurs prior to the elevation of the natriuretic peptide and indicates myocytic damage, which would support significant myocardial injury. Surprisingly, herein, magnetic resonance imaging found no specific evidence of MY despite severe inflammatory syndrome and impaired myocardial function, except in one case who had gadolinium early enhancement in the lateral left ventricular wall, global left ventricle dysfunction by transthoracic ultrasound, ventricular early beats on ECG, and exhibited the highest troponin level at onset $(12123 \mathrm{ng} / \mathrm{L})$. One might argue that troponin levels need to reach some threshold for cardiac resonance magnetic imaging to detect myocardial inflammation. In Luetkens, et al. case report [28], the patient troponine levels were $18,800 \mathrm{ng} / \mathrm{L}$ and CMR imaging showed early gadolinium enhancement due to interstitial myocardial edema.CMR parameters have been defined in the revised Lake Louise criteria for diagnosis of acute myocarditis [29].

The end-diastolic diameter of the left ventricle was not significantly increased in the present study (end-diastolic indexed diameter was within the normal range, i.e. less than $31 \mathrm{~mm} / \mathrm{m}^{2}$ ), which evidences the acute and sudden myocardial injury and absence of myocardial remodeling [9,30-33]. Intravenous immunoglobulins were administered to all patients, and laboratory and ultrasound parameters of systolic function returned to normal in the following days after infusion, i.e. within two weeks after onset of the disease. Inotrope support could be discontinued in less than one week after admission. The recovery of myocardial contractile function was observed very quickly after intravenous immunoglobulins infusion. This favorable and prompt outcome is unusual in pediatric acute MY, where the ability of injured myocardium to improve is uncertain and in any case occurs late and within months after onset of the disease [34]. Interestingly, the data presented herein show impairment of diastolic function in the immediate course after recovery of myocardial contractility and regression of the inflammatory process; one might argue that diastolic dysfunction was present earlier and only revealed after normalization of systolic function. These data encourage to monitor the myocardial cardiological outcome of these patients in the long-term in order to detect further myocardial fibrosis. No report exists to date about the mid and long-term outcomes of myocardium systolic and diastolic function. Further studies are needed to determine the potential of myocardium to evolve towards irreversible scars and fibrosis, by magnetic resonance imaging and/or echocardiographic and Doppler evaluation.

\section{Conclusion}

The present study has found that SARS-CoV-2 myocarditis-like cases are characterized by a more severe laboratory test profile than the Kawasaki-like cases, and has evidenced that cardiac injury is parallel to laboratory test changes. Serial echocardiographic monitoring of systolic and diastolic myocardial parameters should be also needed. Moreover, despite restoration of myocardial contractility, long-term evaluation of diastolic function disorders and detection of fibrosis should be recommended.

\section{Declarations of interest}

None.

\section{References}

1. Riphagen S, Gomez X, Gonzalez-Martinez C, Wilkinson N, Theocharis $P(2020)$ Hyperinflammatory shock in children during COVID-19 pandemic. Lancet 395: 1607-1608.

2. Deza Leon MP, Redzepi A, McGrath E, Abdel-Haq N, Shawaqfeh A, et al. (2020) COVID-19-associated pediatric multi-system inflammatory syndrome. J Pediatric Infect Dis Soc 9: 407-408.

3. Soy $M$, Keser $G$, Atagündüz $P$, Tabak $F$, Atagündüz $I$, et al. (2020) Cytokine storm in COVID-19: Pathogenesis and overview of anti-inflammatory agents used in treatment. Clin Rheumatol 39: 2085-2094.

4. Huang I, Pranata R (2020) Lymphopenia in severe coronavirus disease-2019 (COVID-19): Systematic review and meta-analysis. J Int Care 8: 36.

5. Hennon TR, Penque MD, Abdul-Aziz R, Alibrahim OS, McGreevy MB, et al. (2020) COVID-19 associated Multisystem Inflammatory Syndrome in Children (MIS-C) guidelines; A Western New York approach. Prog Pediatr Cardiol 101232.

6. Molloy EJ, Bearer CF (2020) COVID-19 in children and altered inflammatory responses. Pediatr Res 88: 340-341.

7. Nandy S, Wan SH, Brenes-Salazar J (2020) Cardiovascular manifestations of COVID-19. Curr Cardiol Rev.

8. Kwenandar F, Valeriani Japar K, Damay V, Hariyanto TI, Tanaka M, et al. (2020) Coronavirus disease 2019 and cardiovascular system: A narrative review. Int J Cardiol Heart Vasc 29: 100557

9. Lang JP, Wang X, Moura FA, Siddiqi HK, Morrow DA, et al. (2020). A current review of COVID-19 for the cardiovascular specialist. Am Heart J 226: 29-44.

10. Shaobo Shi, Mu Qin, Bo Shen, Yuli Cai, Tao Liu, et al. (2020) Association of cardiac injury with mortality in hospitalized patients with COVID-19 in Wuhan, China. JAMA Cardiol 5: 802-810.

11. Raul D Mitrani, Nitika Dabas, Jeffrey J Goldberger (2020) COVID-19 cardiac injury: Implications for long-term surveillance and outcomes in survivors. Heart Rhythm 17: 1984-1990.

12. Hendren NS, Drazner MH, Bozkurt B, Cooper Jr LT (2020) Description and proposed management of the acute COVID-19 cardiovascular syndrome. Circulation 141: 19031914. 
13. Xu S, Chen M, Weng J (2020) COVID-19 and kawasaki disease in children. Pharmacol Res 159: 104951.

14. Licciardi F, Pruccoli G, Denina M, Parodi E, Taglietto M, et al. (2020) SARS-CoV-2-induced Kawasaki-like hyperinflammatory syndrome: A novel COVID phenotype in children. Pediatrics 146: e20201711.

15. Viner RM, Whittaker E (2020) Kawasaki-like disease: Emerging complication during the COVID-19 pandemic. Lancet 395: 1741-1743.

16. Battista Calabri G, Formigari R (2020) Covid-19 and kawasaki disease: A glimpse at the past for a predictable future. Pediatr Cardiol 41: 1075.

17. Jones VG, Mills M, Suarez D, Hogan CA, Yeh D, et al. (2020) COVID-19 and Kawasaki disease: Novel virus and novel case. Hosp Pediatr 10: 537-540.

18. Alizargar J (2020) The novel coronavirus (COVID-19) and the risk of Kawasaki disease in children. J Formos Med Assoc 119: 1713-1714.

19. Belhadjer Z, Méot M, Bajolle F, Khraiche D, Legendre A, et al. (2020) Acute heart failure in multisystem inflammatory syndrome in children (MIS-C) in the context of global SARS-CoV-2 pandemic. Circulation 142: 429-436.

20. Driggin E, Madhavan MV, Bikdeli B, Chuich T, Laracy J, et al. (2020) Cardiovascular considerations for patients, health care workers, and health systems during the COVID-19 pandemic. J Am Coll Cardiol 75: 2352-2371.

21. Long B, Brady WJ, Koyfman A, Gottlieb M (2020) Cardiovascular complications in COVID-19. Am J Emerg Med 38: 1504-1507.

22. Mehta P, McAuley DF, Brown M, Sanchez E, Tattersall RS, et al. (2020) COVID-19: Consider cytokine storm syndromes and immunosuppression. Lancet 395: 1033-1034.

23. Mahajan K, Chandra KS (2020) Cardiovascular comorbidities and complications associated with coronavirus disease 2019. Med J Armed Forces India 76: 253-260.

24. Duerr GD, Heine A, Hamiko M, Zimmer S, Luetkens JA, et al. (2020) Parameters predicting COVID-19-induced myo- cardial injury and mortality. Life Sci 260: 118400.

25. Sanna G, Serrau G, Bassareo PP, Neroni P, Fanos V, et al. (2020) Children's heart and COVID-19: Up-to-date evidence in the form of a systematic review. Eur J Pediatr 179: 1079-1087.

26. Madjid M, Safavi-Naeini P, Solomon SD, Vardeny O (2020) Potential effects of coronaviruses on the cardiovascular system: A review. JAMA Cardiol 5: 831-840.

27. Barach P, Lipshultz SE (2020) Rethinking COVID-19 in children: Lessons learned from pediatric viral and inflammatory cardiovascular diseases. Prog Pediatr Cardiol 101233.

28. Luetkens JA, Isaak A, Zimmer S, Nattermann J, Sprinkart AM, et al. (2020) Diffuse myocardial inflammation in COVID-19 associated myocarditis detected by multiparametric cardiac magnetic resonance imaging. Circulation: Cardiovascular Imaging 13

29. Ferreira VM, Schulz-Menger J, Holmvang G, Kramer CM, Carbone I, et al. (2018) Cardiovascular magnetic resonance in nonischemic myocardial inflammation. Expert recommendations. J Am Coll Cardiol 72: 3158-3176.

30. Mahmoud-Elsayed HM, Moody WE, Bradlow WM, KhanKheil AM, Senior J, et al. (2020) Echocardiographic findings in patients with Covid-19 Pneumonia. Can J Cardiol 36: 1203-1207.

31. American College of Cardiology (2020) ACC Clinical Bulletin focuses on Cardiac implications of novel coronavirus (COVID-19).

32. Sala S, Peretto G, Gramegna M, Palmisano A, Villatore A, et al. (2020) Acute myocarditis presenting as a reverse Tako-Tsubo syndrome in a patient with SARS-CoV-2 respiratory infection. Eur Heart J 41: 1861-1862.

33. Clerkin KJ, Fried JA, Raikhelkar J, Sayer G, Griffin JM, et al. (2020) Coronavirus disease 2019 (COVID-19) and cardiovascular disease. Circulation 141: 1648-1655.

34. Ammirati E, Veronese G, Bottiroli M, Wen Wang D, Cipriani M, et al. (2020) Update on acute myocarditis. Trends Cardiovasc Med. 